\title{
Towards self-tuning residual generators for UAV control surface fault diagnosis
}

\author{
Blanke, Mogens; Hansen, Søren
}

Published in:

Proceedings of 2nd International Conference on Control and Fault-Tolerant Systems

Publication date:

2013

Link back to DTU Orbit

Citation (APA):

Blanke, M., \& Hansen, S. (2013). Towards self-tuning residual generators for UAV control surface fault diagnosis. In Proceedings of 2nd International Conference on Control and Fault-Tolerant Systems (pp. 37-42). IEEE.

\section{General rights}

Copyright and moral rights for the publications made accessible in the public portal are retained by the authors and/or other copyright owners and it is a condition of accessing publications that users recognise and abide by the legal requirements associated with these rights.

- Users may download and print one copy of any publication from the public portal for the purpose of private study or research.

- You may not further distribute the material or use it for any profit-making activity or commercial gain

- You may freely distribute the URL identifying the publication in the public portal

If you believe that this document breaches copyright please contact us providing details, and we will remove access to the work immediately and investigate your claim. 


\title{
Towards self-tuning residual generators for UAV control surface fault diagnosis
}

\author{
Mogens Blanke $e^{1,2}$ and Søren Hansen ${ }^{1}$
}

\begin{abstract}
Control surface fault diagnosis is essential for timely detection of manoeuvring and stability risks for an unmanned aircraft. Timely detection is crucial since control surface related faults impact stability of flight and safety. Reliable diagnosis require well fitting dynamical models but with the high cost of detailed modelling and wind tunnel testing, it would be highly desirable if good diagnosis could be obtained with very generic models that are adapted to individual conditions of aircraft and of its operation. This paper presents an approach where a basic generic model is applied and necessary parameters in residual generators are identified on the fly. Initial estimates of parameters are known from off-line analysis of previous flights. The paper analyses how such self-tuning residual generators are combined with change detection to obtain timely fault diagnosis. The paper investigates the parameter convergence and detection properties for the suggested combination of identification and change detection techniques and shows design aspects and trade-offs to be made to make this scheme an effective and robust system for diagnosis or even prognosis. Results are verified using a number of test flights with different members of a population of UAVs that have inherent model uncertainty from one member to another and from one flight to another. Events with actual faults on control surfaces demonstrates the efficacy of the approach.
\end{abstract}

\section{INTRODUCTION}

When models of static and dynamic behaviours are available, fault detection and isolation rely either on generation of parity relations and associated residuals or on estimation of parameters and check of whether parameters exceed given bounds. When models are not available with any satisfactory accuracy, or are just quite uncertain, but a basic model structure is known from first physical principles, self-tuning of detection filters and parameters for change detection would be ideal.

Traditionally fault diagnosis is approached by either parameter identification methods or change detection based on residuals that would ideally be zero in no-fault cases. The two approaches are dealt with in considerable detail in the rich literature on fault diagnosis. Extended Kalman filters that estimate parameters and states could be employed for adaptive fault diagnosis, but success of the EKF techniques depend on knowledge on process and noise characteristics. The innovations formulation [1] and extension to nonlinear

1 M. Blanke and S. Hansen are professor and assistant professor, respectively, in the Automation and Control Group at Dept. of Electrical Engineering, Technical University of Denmark, Elektrovej B. 326, DK-2800 Kgs.Lyngby, Denmark . (mb@elektro.dtu.dk and shaelektro.dtu.dk)

2 M. Blanke is furthermore adjunct professor at the Centre for Autonomous Marine Operations and Systems (AMOS) at Institute of Technical Cybernetics, Norwegian University of Science and Technology, NTNU, NO-7491 Trondheim, Norway cases in [2] dealt with this issue by estimating Kalman gains directly. Particle - and unscented filter approaches have approached state and parameter estimation with success also in non-Gaussian cases, but the algorithms become rather heavy and simpler techniques that could make use of crude models with low number of parameters would be preferred if they at the same time provide robust schemes for diagnosis. A direct combination of parameter estimation and change detection in a self-tuning scheme appears appealing. A combined treatment of adaptive methods and change detection was provided in [3] and references herein. An approach that combines parameter identification and residual evaluation was presented in [4] where an analytic design was achieved of a robust fault detection scheme, that takes both the noise in the online measurements as well as the identification uncertainty into account. However, the assumption here was the fault detection was considered a residual generation and evaluation problem aiming at additive faults.

With the wide penetration of autonomous technologies, very visible in unmanned aerial vehicles (UAV), fault diagnosis and remedial actions to faults become essential for safety. This paper therefore exemplifies a self-tuning diagnostic approach on critical actuator and control surface faults on UAVs. Extensive research has been conducted for UAVs related to control surface faults and failures. These include [5] and [6] where the focus was on how faults directly affect the UAV's ability to maneuver. Reconfigurations of the control and guidance systems were done to accommodate the reduced capabilities of the aircraft. The FDI was achieved by using an extended version of multiple model adaptive estimation (MMAE). The faults were modelled as an unknown signal controlling the actuators, which were then estimated by extended Kalman filters in the enhanced MMAE method. A structural analysis approach to diagnosis was considered in [7] where additive faults were diagnosed on sensors and actuators. In [8] FTC for a small UAV subject to control surface faults was investigated. The authors estimated the reduction of UAV's flight envelope and used active FDI to isolate faults on the control surfaces. Methods using linear parameter varying (LPV) methods were dealt with in [9]. The relevance for manned aircraft is obvious and was treated in [10], [11],[12].

This paper considers the possibilities of arriving at a selftuning scheme for diagnosis and exemplifies the findings with thorough analysis of data from aerial vehicles in cases without and with faults or failures in control surfaces. The issues considered include: the choice between detection of change of parameters or change in residuals or a combination 
of both; assessment of model order and of normal ranges of parameters; selection of change detection method and thresholds for hypothesis testing with specified false alarm probability; dealing with non-ideal signal properties, not Gaussian and not uncorrelated. This paper is a generalisation and unified treatment of first approaches presented in [13] and [14]. The paper considerers an UAV with ailerons and elevators for control, a rudder is not present. Records from actual flights are used for illustration of the self-tuning approach.

\section{CONTROL SURFACE FAUlT MODEL}

The common first-principles model of an aircraft gives the well known 6 degrees of freedom dynamic and kinematic description. To facilitate this model knowledge about force and torque coefficients must be obtained for the involved aircraft and this information is not always available for cheaper UAV's. For control surface fault diagnosis the important feature is the relationship between surface deflection and angular rates. Using $p, q, r$ for body rates in roll, pitch and yaw, respectively, and control surface deflections $\delta_{a}$ for ailerons and $\delta_{e}$ for elevators, dynamic relations take the following generic form in a linear parameter varying model with air speed $U$ being the dominating parameter. A discrete time ARX representation is,

$$
\begin{aligned}
A_{p a}(U) p[k] & =B_{p a}(U) \delta_{a}[k] \\
A_{q e}(U) q[k] & =B_{q e}(U) \delta_{e}[k] \\
A_{r a}(U) r[k] & =B_{r a}(U) \delta_{a}[k],
\end{aligned}
$$

where $A$ and $B$ are polynomials in the discrete time delay operator. With air speed a dominant parameter in the LPV model, air speed system fault diagnosis is essential. The was the topic dealt with in [15].

\section{A. Rough model for diagnosis}

When actuator faults occur, the steady properties of Eq. (1) are mainly affected. A static model in $p$ and $q$, with AX structure in $r$, Eqs. (2) to (4), where $b_{p a}, b_{q e}$ and $c_{r a}$ are bias terms and $a_{p a}, a_{q e}$ and $a_{r a}$ are gain factors, hide literally all dynamics except for the integrating effect between the aileron and yaw rate in the $b_{r a}$ term,

$$
\begin{aligned}
p[k] & =a_{p a}(U) \delta_{a}[k]+b_{p a} \\
q[k] & =a_{q e}(U) \delta_{e}[k]+b_{q e} \\
r[k] & =a_{r a}(U) \delta_{a}[k]+b_{r a}(U) r[k-1]+c_{r a} .
\end{aligned}
$$

This approach separates the lateral and longitudinal states since the aileron is only related to roll and yaw and the elevator is related to pitch.

Equations (2) to (4) can be described on the form,

$$
y[k]=\boldsymbol{\varphi}[k]^{\mathrm{T}} \boldsymbol{\Theta}[k]+e[k],
$$

with $e[k]$ being the un-modelled behaviour. For (2) the parameters would be:

$$
\begin{aligned}
y[k] & =p[k] \\
\boldsymbol{\varphi}[k] & =\left[\begin{array}{c}
\delta_{a}[k] \\
1
\end{array}\right] \\
\boldsymbol{\Theta}[k] & =\left[\begin{array}{c}
a_{p a} \\
b_{p a}
\end{array}\right] .
\end{aligned}
$$

To estimate parameters online, a recursive least squares (RLS) is a simple choice with the following recursive implementation,

$$
\begin{aligned}
\boldsymbol{\varepsilon}[k] & =y[k]-\boldsymbol{\varphi}[k]^{\mathrm{T}} \hat{\boldsymbol{\Theta}}[k-1] \\
P[k] & =\left(\lambda_{f} P[k-1]^{-1}+\boldsymbol{\varphi}[k] \boldsymbol{\varphi}[k]^{\mathrm{T}}\right)^{-1} \\
\hat{\boldsymbol{\Theta}}[k] & =\hat{\boldsymbol{\Theta}}[k-1]+P[k] \boldsymbol{\varphi}[k] \boldsymbol{\varepsilon}[k] .
\end{aligned}
$$

In this, $\lambda_{f}$ is the forgetting factor and $P[k]$ is the estimator's covariance. The initial value of $P[k]$ can be determined from some average of test flights: running the estimator for data from steady wings-level flight without faults would give an a-priori value of $P[k]$. Alternatively, on-line estimation would be possible using standard techniques [16].

The forgetting factor is tunable and this is one of the parameters to be considered when combining parameter identification with residual evaluation for diagnosis. Control surface defects will give rise to rapid change in the input/output signals and hence in the prediction error (9) and subsequently appear as a parameter adaptation to the faulty case.

\section{B. Properties of residuals and parameter identification}

Three residuals are formed based on (9), the prediction error between measurements and estimates. The individual residuals employ $R_{p a}$ from (2), $R_{q e}$ from (3) and $R_{r a}$ from (4),

$$
\begin{aligned}
R_{p a} & =p[k]-a_{p a} \delta_{a}[k]-b_{p a} \\
R_{q e} & =q[k]-a_{q e} \delta_{e}[k]-b_{q e} \\
R_{r a} & =r[k]-a_{r a} \delta_{a}[k]-b_{r a} r[k-1]-c_{r a} .
\end{aligned}
$$

The development over time for these three residuals is shown in Fig. 1 for a flight where an aileron fault developed and caused the loss of the UAV.

\section{Distribution of residuals}

When residuals generated for UAV's are sampled at frequencies below $10 \mathrm{~Hz}$ (here $5 \mathrm{~Hz}$ ), fast dynamics is not captured and the source of uncertainty is not only wind and measurement noise but also the result of motion in a normal manoeuvring pattern. This causes the distribution of residuals to deviate from Gaussian or Gaussian mixture. By running a Kolmogorov-Smirnov test on the residuals it was found that they follow the Laplacian distribution given by.

$$
p(x ; a, b)=\frac{1}{2 b} \exp \left(-\frac{|x-a|}{b}\right)
$$

where $a$ is the median and $b$ is the scaling. The distribution of residuals has an impact on the derivation later on of change detector. 

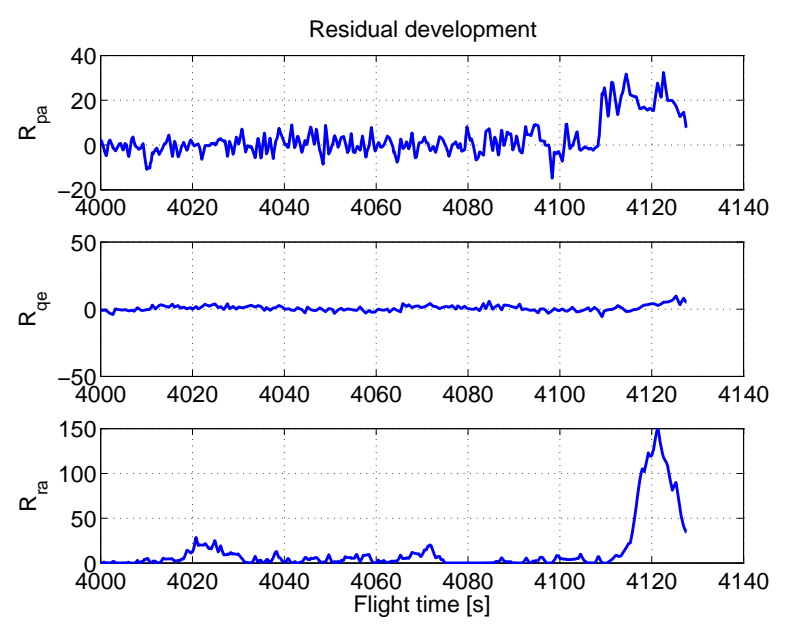

Fig. 1. Time development of the three residuals from a flight where an aileron fault developed shortly before mission aborted.

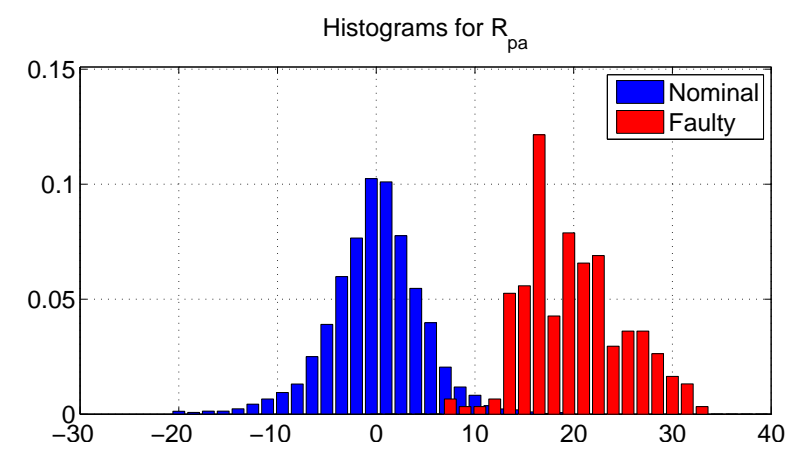

Fig. 2. Histogram of raw residual Eq. (14) before and after fault on aileron Calculated from flight data.

\section{Correlation structure}

In many real-life cases, and also the one at hand, raw residuals are far from white, as seen from the power spectrum and autocorrelation in Fig. 3.
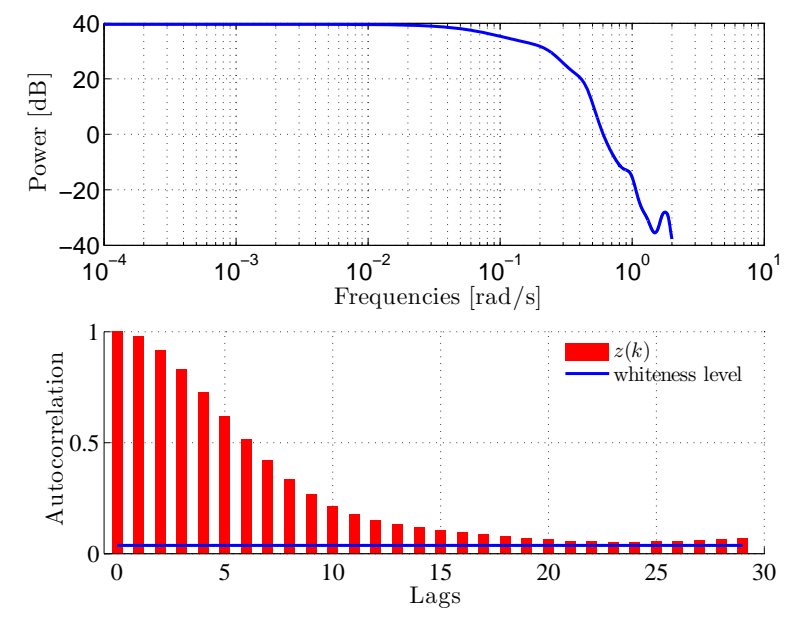

Fig. 3. Power spectrum and autocorrelation for raw residual Eq. (14).

Discrete time residuals are required to meet a condition that samples are independent and identically distributed (IID) in order that standard theoretical results for the commonly adopted Neyman-Pearson test (log likelihood test over a window of observations) hold for distribution of test statistic and thereby for threshold determination, false alarm probability and probability of detection, see eg. [17].

The Neyman-Pearson test distinguish hypothesis $\mathscr{H}_{1}$ (fault, associated with parameter vector $\theta_{1}$ ) from hypothesis $\mathscr{H}_{0}$ (no fault, associated with parameter vector $\theta_{0}$ ) from a vector of observations $\mathbf{x}$

$$
\frac{p\left(\mathbf{x} ; \theta_{1}\right)}{p\left(\mathbf{x} ; \theta_{0}\right)}>\gamma
$$

With distribution of individual observations $x[k]$ being $p\left(x[k] ; \mathscr{H}_{0}\right)$, the distribution of the vector of observations is

$$
p\left(\mathbf{x} ; \mathscr{H}_{0}\right)=\prod_{k=1}^{N} p\left(x[k] ; \mathscr{H}_{0}\right)
$$

only if the IID condition is met. Otherwise, conditional probabilities $p\left(x[k] \mid x[k-1], \ldots ; \mathscr{H}_{0}\right.$ enter into the calculation of $p\left(\mathbf{x} ; \mathscr{H}_{0}\right)$ and make the distribution calculable for Gaussian cases but not for general distributions.

\section{E. Whitening}

Whitening of residuals is a standard procedure but it contains uncertain elements. If a discrete time scalar residual $r_{i}[k]$ was generated through an auto regressive moving average (ARMA) model by a scalar process $e[k]$ with independent increments,

$$
r_{i}[k]=\frac{C\left(z^{-1}\right)}{F\left(z^{-1}\right)} e[k]
$$

where $F\left(z^{-1}\right)$, and $C\left(z^{-1}\right)$ are polynomials in the delay operator $z^{-1}$, then, provided $C\left(z^{-1}\right)$ is Hurwitz, the original residual, $r_{i}(k)$ for brevity, would be whitened to $r_{i}^{w}[k]$ by

$$
r_{i}^{w}[k]=\frac{F\left(z^{-1}\right)}{C\left(z^{-1}\right)} r_{i}[k]
$$

Standard identification methods can be used to determine the polynomials in the ARMA model Eq. (18). However, $C\left(z^{-1}\right)$ need be restricted to have eigenvalues within the unit circle and it would be desirable to be able to avoid whitening.

\section{HYPOTHESIS TESTING}

Two set of measures are available for hypothesis testing, the test statistics based on non IID residuals and the estimates of parameters.

\section{A. Change detection based on GLRT test statistic}

Since the magnitude of change are not known, changes in the three residual signals are detected using the Generalised Likelihood Ratio Test (GLRT). The nominal case, $\mathscr{H}_{0}$, for the residuals is when their median value $a$ is close to zero. A median different from zero indicates a discrepancy between the aircraft model and the measurements, which in turn indicates a fault. Fig. 2 shows histograms of residuals based on flight actual without and with a fault. 
The two hypothesis for fault detection are,

$$
\begin{aligned}
& \mathscr{H}_{0}: a=0 \\
& \mathscr{H}_{1}: a \neq 0
\end{aligned}
$$

A test statistic for the Laplacian distribution for changes in the median $a$, can be formulated as follows,

$$
T_{L}(\boldsymbol{x})=N \log \left(\frac{\hat{b}_{0}}{\hat{b}_{1}}\right)>\gamma
$$

The form of GLR detector is fixed once the overall problem has been fixed, i.e. the self-tuning does not include choice of GLR detector. The essential parameter, the window size $M$ in the GLR detector is, however, included as a parameter subject to self-tuning. The exact form of the GLRT for Laplacian distribution was derived in [18].

\section{B. Threshold selection for test statistic}

When the IID condition is not met, the threshold value $\gamma$ can still be chosen from false alarm and detection probabilities. The threshold $\gamma$ is selected to obtain a sufficiently low false-alarm rate $P_{F A}$ with the Weibull distribution of the GLRT statistic as:

$$
P_{F A}=\int_{g: L_{G}(\mathbf{z}(k))>\gamma} p_{W}\left(g ; \mathscr{H}_{0}\right) d g ;
$$

where $p_{W}\left(g ; \mathscr{H}_{0}\right)$ is the PDF of the Weibull distribution for the GLRT statistics under $\mathscr{H}_{0}$.

Estimation of the Weibull parameters $v_{0 g}$ and $\beta_{0 g}$ is obtained under $\mathscr{H}_{0}$ as $p_{W}\left(g ; \mathscr{H}_{0}\right)$. The threshold for a desired (low) false alarm probability $P_{F A}$ is then obtained from,

$$
1-P_{F A}=1-\exp \left(-\left(\frac{\gamma}{v_{o g}}\right)^{\beta_{0 g}}\right)
$$

Then the threshold $\gamma$ is,

$$
\gamma=v_{0 g}\left(-\ln P_{F A}\right)^{\frac{1}{\beta_{0 g}}}
$$

Fig. 4 shows a probability plot of data for flights without faults and an approximating distribution, the parameters of which are easily calculated by standard methods. The threshold is determined from the approximating function extrapolating into the tail of data.

\section{Change detection based on parameter estimates}

The parameter estimation over time of a mission is somewhat dependent on properties of the aerial vehicle itself. As a simple effect, fuel consumed during the flight may change the centre of gravity of the vehicle and require torque compensation by an offset in elevator angle that develops enroute. Histograms of the parameter estimates enroute the selected test flight are shown in Fig. 5. Data after the aileron fault develops are shown in red.

Common approaches to alarm levels for parameter deviation form normal are to trigger an alarm if a parameter deviated more than 3 to $6 \sigma$ from its mean value. If choosing this approach, the aileron fault would clearly be detected, but

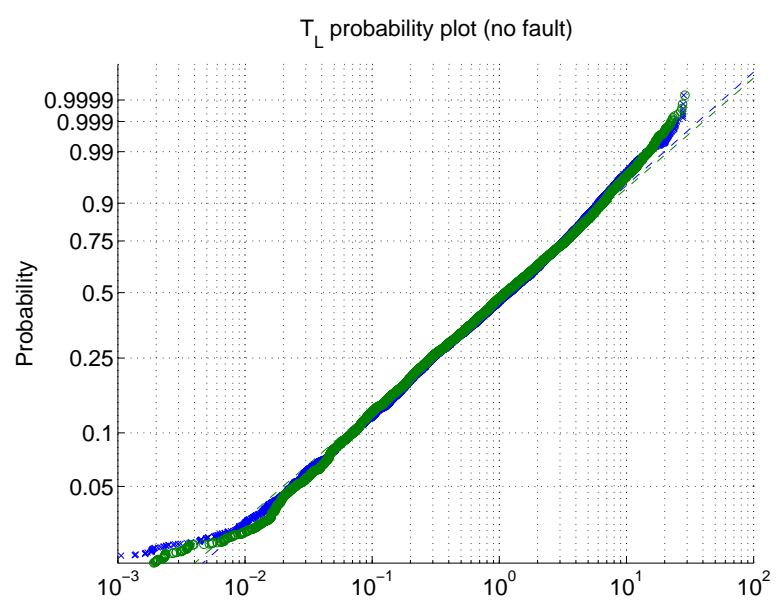

Fig. 4. Probability plot for test statistic, $T_{L}$, for flights of aircraft with no faults. The dashed line is the estimated distribution.
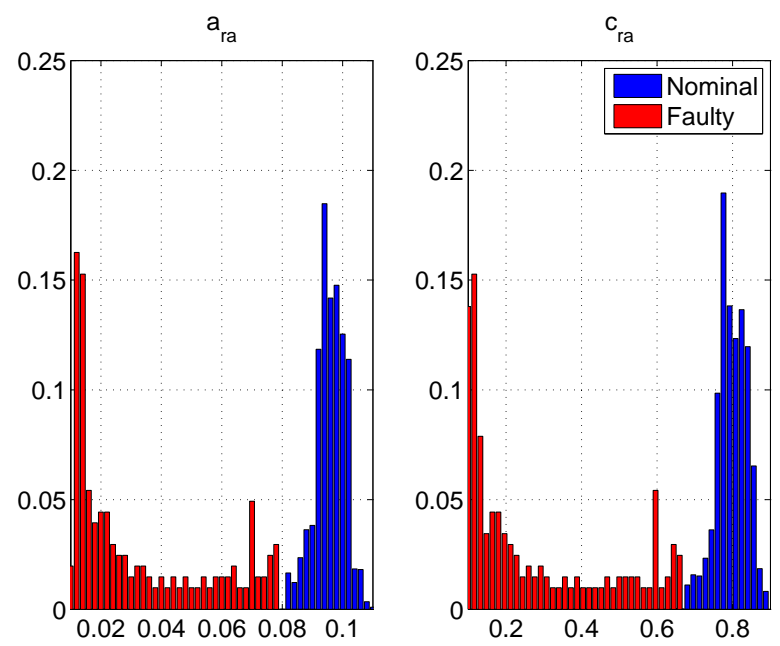

Fig. 5. Histograms for parameter estimates. Blue is $\mathscr{H}_{0}$, red is $\mathscr{H}_{1}$ for aircraft with an aileron fault.

detection probability could clearly be better as there would be a noticeable amount of $\left\langle_{1}\right.$ data within the threshold limits.

Fig. 6 shows the development of parameter estimates before and during development of an aileron failure. Fig. 7 shows parameter convergence and confidence intervals during no-fault operation.

\section{Classification based on joint probability}

Combining the two detectors provide the false alarm probability,

$$
\begin{aligned}
P_{F A}\left(g,{ }^{f} \mathscr{S}_{\phi^{2} \theta}\right) & \triangleq P_{F A}\left(g>\gamma_{g} \cap{ }^{f} \mathscr{S}_{\phi^{2} \theta}>\overline{\mathscr{S}}\right) \\
& =P_{F A}\left(g>\left.\gamma_{g}\right|^{f} \mathscr{S}_{\phi^{2} \theta}>\overline{\mathscr{S}}\right) P_{F A}\left({ }^{f} \mathscr{S}_{\phi^{2} \theta}>\overline{\mathscr{S}}\right) .
\end{aligned}
$$

The effect of combined hypothesis testing can be a significantly reduced false alarm probability. If $P_{F A}\left(g>\gamma_{g} \mid{ }^{f} \mathscr{S}_{\phi^{2} \theta}>\overline{\mathscr{S}}\right) \simeq P_{F A}\left(g>\gamma_{g}\right), \quad P_{F A}\left(g,{ }^{f} \mathscr{S}_{\phi^{2} \theta}\right) \simeq$ $P_{F A}\left(g>\gamma_{g}\right) P_{F A}\left({ }^{f} \mathscr{S}_{\phi^{2} \theta}>\overline{\mathscr{S}}\right)$, but data are not available to make this conclusion at a rigourous level. At a heuristic 

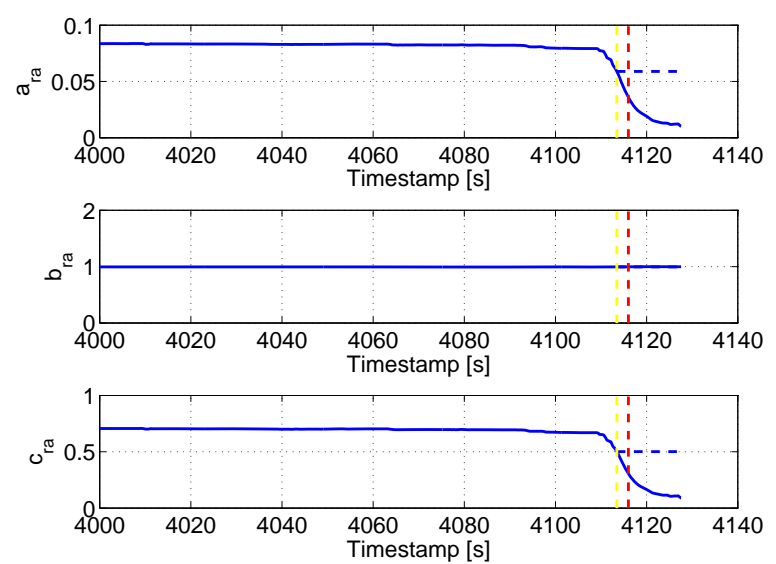

Fig. 6. Parameters estimates for $R_{r a}$ when a fault occurs. The fault is detected at $T=4116 \mathrm{~s}$. The parameter estimation is usually stopped when a fault is detected, but to illustrate the progress the estimation is continued here.
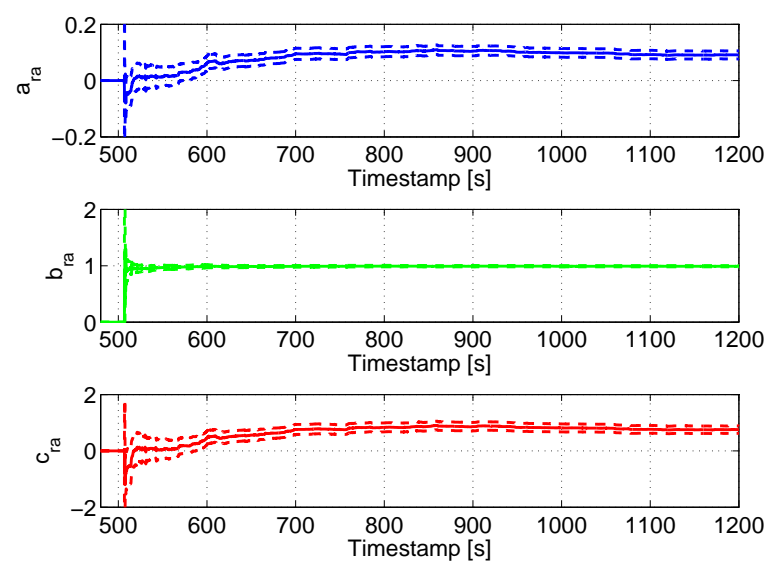

Fig. 7. Parameters estimates for $R_{r a}$ in normal flight conditions. The $95 \%$ confidence boundaries are indicated with the dashed lines.

level, however, a significant improvement of the use of the combined detectors is illustrated in Fig. 8 for the data set. It is clearly much easier to distinguish between the $\mathscr{H}_{0}$ and ${ }_{1}$ cases when making the hypothesis based on both parameter and residual evaluation than on any of the two as individual tests.

Selecting thresholds for the combined parameter and residual evaluation ideally should require consideration of detection probability as well,

$$
P_{D}\left(g,{ }^{f} \mathscr{S}_{\phi^{2} \theta}\right)=\int_{\mathscr{I}} p\left(g,{ }^{f} \mathscr{S}_{\phi^{2} \theta} ; \mathscr{H}_{1}\right) \mathrm{d} g \mathrm{~d}^{f} \mathscr{S}_{\phi^{2} \theta},
$$

where $\mathscr{I}=\left\{g: g \geq \gamma_{g}\right\} \cap\left\{{ }^{f} \mathscr{S}_{\phi^{2} \theta}:{ }^{f} \mathscr{S}_{\phi^{2} \theta} \geq \overline{\mathscr{S}}\right\}$. This computation would need the joint distributions for behaviours under normal conditions with the natural variation of parameters and when faults occur. A real failure was present in the data used as an example in this paper, but further study is required of behaviours of different types of faults before being able to make conclusive statements about detection probabilities for real life cases of faults.
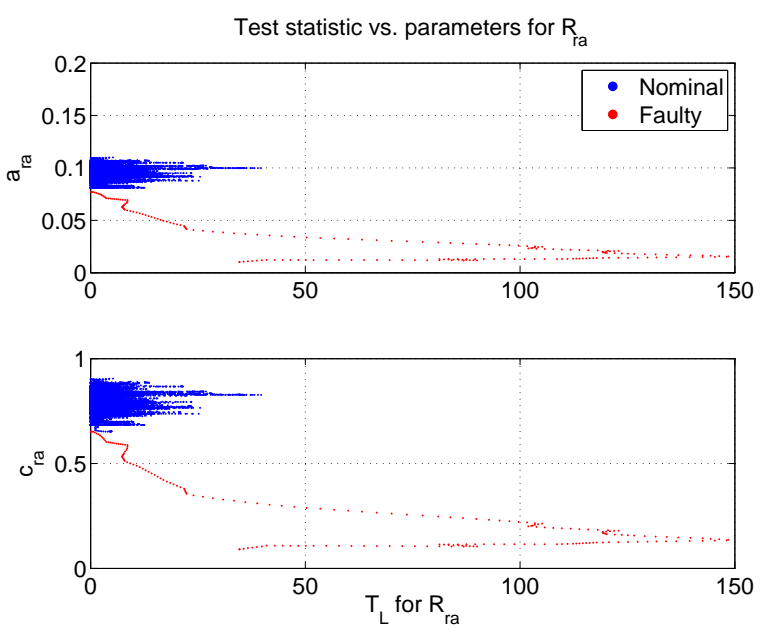

Fig. 8. Scatter diagram of test statistic, $T_{L}$, versus parameter estimates. Blue is $\mathscr{H}_{0}$, red is $\mathscr{H}_{1}$ for aircraft with an aileron fault.

\section{RESUlts}

Fig. 9 shows the time development of the test statistic for the selected flight. The threshold levels are indicated by the dashed line. This line is crossed at around $4120 \mathrm{~s}$ for both $R_{p a}$ and $R_{r a}$ due to an aileron fault on the aircraft.

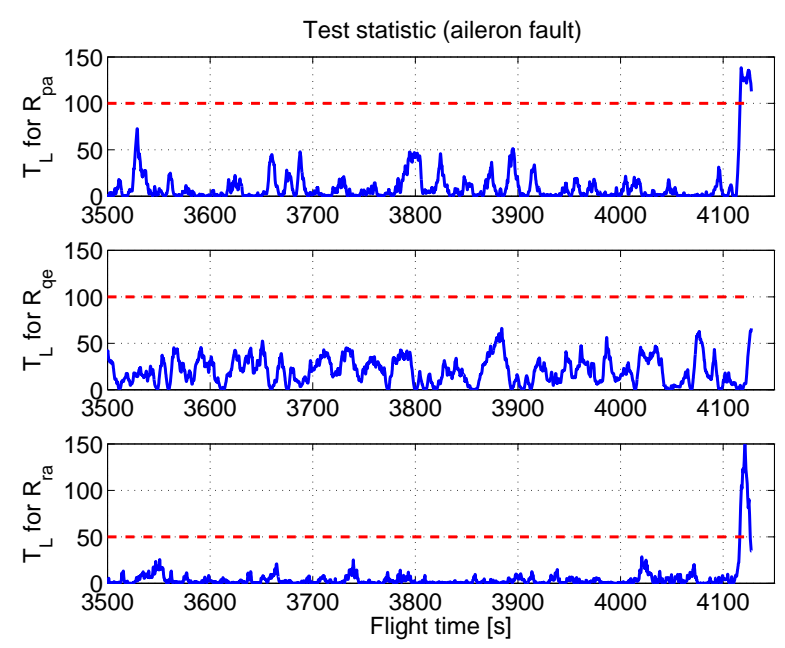

Fig. 9. Time development of test statistic, $T_{L}$, for aircraft with an aileron fault.

Fig. 10 shows the result of combined detection using both violation of parameter window and overrun of GLRT threshold. Requiring that both criteria are fulfilled generates zero false alarms on the test data set and it detects and alerts faster than any of the individual (single source) hypothesis tests.

The result of classification for hypothesis testing using the combined tests for change of parameter and the change in the GLR test statistic provide very promising results.

\section{CONCLUSIONS}

This paper demonstrated some steps towards self-tuning fault diagnosis and exemplified the approach by control 

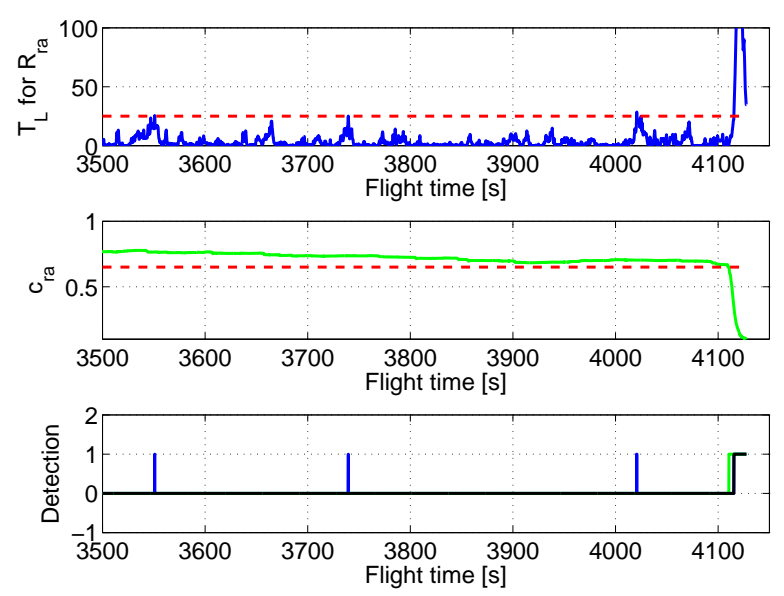

Fig. 10. GLRT test statistic and parameter estimates versus time and alarms generated by exceeding thresholds in a: test statistics from residual, b: parameter estimate deviates, c: combined test where both (a) and (b) are fulfilled simultaneously. The combined testing generates zero false alarms and detects earlier than any of the individual tests.

surface fault diagnosis for a particular type of unmanned aerial vehicle. Data from these UAVs in regular service illustrated the methods with cases where control surface defects developed in the air.

The paper showed how self-tuning could be employed to obtain adaptation to flight parameters while retaining the capability to detect faults. Change detection was applied to residuals alone, and to residuals combined with parameter estimates. The combination of the two tests appeared to possess superior quality with respect to classification and early detection. The threshold used for change detection was estimated to obtain a desired false alarm probability, the threshold was determined through estimation of parameters in the distribution of the test statistics. The approach appeared quite promising indeed from assessments made on real flight data.

\section{ACKNOWLEDGEMENTS}

The support from the Danish Forces Joint UAV Team and the permission to publish flight data in this paper is greatly appreciated. The authors also wish to acknowledge the collaboration with Meggitt Defence Systems, UK, for providing access to telemetry data protocols.

\section{REFERENCES}

[1] L. Ljung, "Asymptodic behavior of the extended kalman filter as a parameter estimator for linear systems," IEEE Trans. of Automatic Control, vol. 29 (1), pp. 36-50, 1979.

[2] W.-w. Zhou and M. Blanke, "Identification of a class of non-linear state space models using rpe techniques," IEEE Transactions of Automatic Control, vol. 34 (3), pp. 312-316, 1989.

[3] F. Gustafsson, Adaptive Filtering and Change Detection. Wiley, 2001 (2nd ed.).

[4] J. Dong, M. Verhaegen, and F. Gustafsson, "Robust fault detection with statistical uncertainty in identified parameters," IEEE Transactions on Signal Processing, vol. 60, pp. 5064-5076, 2012.

[5] G. Ducard and H. P. Geering, "Efficient nonlinear actuator fault detection and isolation system for unmanned aerial vehicles," Journal of Guidance, Control, and Dynamics, vol. 31 (1), pp. 225-237, 2008.

[6] G. J. J. Ducard, Fault-tolerant Flight Control and Guidance Systems. Springer Verlag, 2009.

[7] M. L. Fravolini, V. Brunori, G. Campa, M. R. Napolitano, and M. La Cava, "Structured analysis approach for the generation of structured residuals for aircraft fdi," IEEE Transactions on Aerospace and Electronic Systems, vol. 45 (4), pp. 1466-1482, 2009.

[8] F. Bateman, H. Noura, and M. Ouladsine, "Fault diagnosis and faulttolerant control strategy for the aerosonde uav," IEEE Transactions on Aerospace and Electronic Systems, vol. 47, no. 3, pp. 2119-2137, 2011.

[9] D. Henry, A. Zolghadri, J. Cieslak, and D. Efimov, "A lpv approach for early fault detection in aircraft control surfaces servo-loops," in IFAC Proceedings Volumes (IFAC Safeprocess 2012), no. 8, 2012, pp. 808-811.

[10] P. Goupil, "Airbus state of the art and practices on fdi and ftc in flight control system," Control Engineering Practice, vol. 19 (6), pp. 524 539, 2011.

[11] E. Alcorta-Garcia, A. Zolghadri, and P. Goupil, "A nonlinear observerbased strategy for aircraft oscillatory failure detection: A380 case study," IEEE Transactions on Aerospace And Electronic Systems, vol. 47 (4), pp. 2792-2806, 2011.

[12] A. Zolghadri, "Advanced model-based fdir techniques for aerospace systems: Today challenges and opportunities," Progress in Aerospace Sciences, vol. 53, pp. 18-29, 2012.

[13] S. Hansen and M. Blanke, "Control surface fault diagnosis with specified detection probability - real event experiences," in The 2013 International Conference on Unmanned Aircraft Systems, 2013.

[14] —, "In-flight fault diagnosis for autonomous aircraft via lowrate telemetry channel," in $8^{\text {th }}$ IFAC Int. Symp. on Fault Detection, Supervision and Safety for Technical Processes, 2012.

[15] — - "Diagnosis of airspeed measurement faults for unmanned aerial vehicles," IEEE Transac. Aerospace and Electronic Systems, 2013.

[16] L. Ljung and T. Söderström, Theory and Practice of Recursive Identification. MIT Press, 1983.

[17] S. M. Kay, Fundamentals of Statistical Signal Processing: Detection Theory. Prentice-Hall PTR, 1998.

[18] S. Hansen, M. Blanke, and J. Adrian, "Diagnosis of uav pitot tube defect using statistical change detection," in Proc. 7th IFAC Symposium on Intelligent Autonomous Vehicles, IAV2010,, 2010. 